\title{
A soluble truncated tau species related to cognitive dysfunction and caspase- 2 is elevated in the brain of Huntington's disease patients
}

Peng Liu ${ }^{1,3^{*}}$ (D), Benjamin R. Smith ${ }^{1,3}$, Eric S. Huang ${ }^{1,3,8}$, Abhishek Mahesh ${ }^{5}$, Jean Paul G. Vonsattel ${ }^{6}$, Ashley J. Petersen ${ }^{4}$, Rocio Gomez-Pastor ${ }^{2}$ and Karen H. Ashe , $^{1,2,3,7^{*}}$

\begin{abstract}
Huntington's disease (HD) is a progressive neurodegenerative disease. Involuntary movements, cognitive impairment and psychiatric disturbance are the major clinical manifestations, and gradual atrophy and selective neuronal loss in the striatum and cerebral cortex are the pathologic hallmarks. HD is caused by expanded CAG trinucleotide repeats at the N-terminus of IT15 that encodes the huntingtin (HTT) protein, though the molecular mechanisms through which the mutant $\mathrm{HTT}(\mathrm{mHTT})$ exerts toxic effects remain obscure. Members of the caspase family, including caspase-2 (Casp2), play an important role in HD pathogenesis. Genetic ablation of Casp2 ameliorates cognitive and motor deficits of HD mice, though the molecular targets of Casp2 are still unclear. It is well established that the microtubule-associated protein tau potentiates cognitive dysfunction in a variety of neurodegenerative disorders, including HD. Our recent study indicates that Casp2-catalyzed tau cleavage at aspartate 314 (tau 2N4R isoform numbering system) mediates synaptotoxicity, cognitive deficits and neurodegeneration in cellular and mouse models of frontotemporal dementia; further, levels of $\Delta$ tau314, the soluble, N-terminal cleavage product, are elevated in individuals with mild cognitive impairment and Alzheimer's disease, compared with cognitively normal individuals. Here, we identified the presence of $\Delta$ tau314 proteins in the striatum (caudate nucleus) and prefrontal cortex (Brodmann's area 8/9) of human subjects, and showed that in both structures, levels of Casp2 and $\Delta$ tau314 proteins correlate well, and both proteins are higher in HD patients than non-HD individuals. Our findings advance our understanding of the contribution of Casp2-mediated $\Delta$ tau314 production to HD pathogenesis.
\end{abstract}

Keywords: Microtubule-associated protein tau, Caspase-2, Huntingtin, Frontal cortex, Caudate nucleus, Striatum, Huntington's disease, Immunoprecipitation, Western blotting, Truncation

\section{Introduction}

Huntington's disease (HD) is an autosomal-dominant and progressive brain disorder. The clinical manifestations of HD involve uncontrolled movements, psychiatric disturbance and cognitive decline (for review, see for example [39]); and HD is pathologically characterized by the gradual atrophy of and selective neuronal loss in the neostriatum (caudate nucleus and putamen) and cerebral

\footnotetext{
*Correspondence: liuxx726@umn.edu; hsiao005@umn.edu

${ }^{1}$ Departments of Neurology, University of Minnesota, Minneapolis, MN 55455, USA

Full list of author information is available at the end of the article
}

cortex (for review, see for example [33]). HD is caused by an abnormal expansion of CAG trinucleotide repeats at the N-terminus of IT15 (interesting transcript 15) encoding the huntingtin (HTT) protein [15]. However, the molecular mechanisms through which the mutant HTT (mHTT) leads to the onset and development of HD are not fully understood (for review, see for example [18]).

Cysteine-dependent aspartate-directed proteases (caspases) play an important role in HD pathogenesis. Hydrolysis of mHTT by caspases-2, -3 and $-6[41,42]$ promotes the aggregation of its $\mathrm{N}$-terminal cleavage products in the brain $[10,12,14,19]$. Interestingly, while 
caspase-2 (Casp2) cleaves mHTT, blockage of Casp2mediated mHTT cleavage did not affect disease progression [12]. Yet, genetic ablation of Casp2 led to amelioration of cognitive and motor dysfunctions of transgenic mice overexpressing IT15 with expanded CAG trinucleotides [8]. Thus, it appears that Casp2 mediates the behavioral abnormalities. However, the substrate, whose cleavage by Casp2 leads to the abnormalities, is unknown.

The microtubule-associated protein tau (MAPT) is a highly soluble, unstructured molecule that is primarily present in the neurons of the central nervous system. The contribution of tau to HD pathogenesis involves, but is not limited to, the presence of aggregated tau inclusions in the brain of HD patients [7, 9, 11, 17, 23, 25, 26, 31, 38], the influence of MAPT haplotype in cognition of $\mathrm{HD}$ patients (patients with the $\mathrm{H} 2$ haplotype are more severely cognitively impaired than those with the $\mathrm{H} 1$ haplotype) [38], the amelioration of behavioral abnormalities of HD mice by genetic ablation of Mapt [11], and the mHTTmediated alterations in tau splicing isoform expression [11] and tau hyperphosphorylation [6, 13]. Despite these findings, the exact tau species and the molecular mechanisms through which tau mediates HD pathogenesis are still largely unknown. The association of tau with cognitive dysfunction has been well established in a variety of mouse lines modeling multiple neurodegenerative diseases, and tau proteolysis and acetylation were shown to mediate cognitive impairment of animals [24, 32, 35, 43, 44].

Of particular note, Casp2-mediated tau cleavage at amino acid residue aspartate 314 (D314) (tau 2N4R isoform numbering system hereafter unless specified) is responsible for synaptic function impairment and cognitive deficits in cellular and transgenic mouse models of frontotemporal dementia and parkinsonism linked to chromosome 17 [44], indicating a toxic partnership between Casp2 and tau [36]. In addition, $\Delta$ tau314 proteins, the soluble cleavage products that are immunoreactive to monoclonal tau-13 (a.a. 15-25), were shown to be related to Alzheimer's disease (AD), as the levels of $\Delta$ tau314 proteins are elevated in the disease-affected inferior temporal gyrus region of individuals with mild cognitive impairment (MCI) and $\mathrm{AD}$ patients compared to cognitively normal, elderly individuals [44].

In this study, we identified the presence of $\Delta$ tau314 proteins in two HD-affected brain regions (caudate nucleus and prefrontal cortex (Brodmann's area (BA) 8/9)) of human subjects using conventional immunoprecipitation (IP)/Western blotting (WB) analysis. We showed that HD patients have higher levels of $\Delta$ tau314 proteins as well as Casp2 than non-HD individuals. We also showed that levels of Casp2 and $\Delta$ tau314 proteins are highly correlated. Results of this study, together with previous findings, suggest that Casp2-mediated tau cleavage to produce $\Delta$ tau 314 is a common pathologic mechanism that leads to synaptotoxicity in multiple neurodegenerative diseases.

\section{Materials and methods}

In vitro synthesis of human full-length tau and $\Delta$ tau314 proteins

Complementary DNA (cDNA) encoding the full-length tau $0 \mathrm{~N} 4 \mathrm{R}$ isoform was cloned into the pcDNA3.1(+) mammalian expression vector (Thermo Fisher Scientific, Waltham, MA). For synthesis of the truncated human tau protein $\Delta$ tau314, the trinucleotides encoding amino acid residue leucine 315 were modified to a stop codon using the QuikChange II Site-Directed Mutagenesis Kit (Agilent Technologies, Santa Clara, CA) following the manufacturer's instructions. The following primers were used for the mutagenesis: forward primer, 5' - AGTC TACAAACCAGTTGACTAGAGCAAGGTGACCTCCAAG - 3'; reverse primer, 5' - CTTGGAGGTCACCTTGCTCTAGTCAACTGGTTTGTAGACT - 3'. Protein synthesis was performed using the TnT Coupled Reticulocyte Lysate Systems (Promega, Madison, WI) following the manufacturer's instructions.

\section{Human brain collection}

For the small cohort pilot study, frozen post-mortem specimens of dorsolateral prefrontal cortex (BA 8) from ten de-identified subjects-five HD patients and five non-HD individuals-were obtained from the Institute of Neurobiology Brain Bank (HUB-ICO-IDIBELL Biobank), Bellvitge University Hospital, Hospital del Llobregat, Spain.

For the large cohort study, frozen post-mortem specimens were obtained from the National Institutes of Health (NIH) NeuroBioBank and the New York Brain Bank (NYBB) at Columbia University, New York City, New York. Dorsolateral prefrontal cortex (BA 8) and caudate nucleus tissue from twenty-four de-identified subjects were provided by the NIH NeuroBioBank, and dorsolateral and medial prefrontal cortex (BA 9) tissue from twenty de-identified subjects were provided by NYBB. Tissue specimens from thirteen HD patients and fourteen non-HD individuals (see Additional file 1: Table S1 for detailed demographic characteristics) were used in the study based on the following criteria: 1) the age at death, sex distribution and post-mortem interval (PMI) of brain tissue harvest of the HD patients are not significantly different from those of the non-HD individuals; 2 ) The ages of subjects are 50 years or older, and 3) the PMIs of brain tissue harvest are less than $22 \mathrm{~h}$.

Frozen tissue was stored at $-80^{\circ} \mathrm{C}$ in either polypropylene bags or cryotubes prior to shipment to the University of Minnesota, Twin Cities, Minnesota.

All procedures were approved by the Institutional Review Boards (IRBs) of the HUB-ICO-IDIBELL Biobank (Spain), 
the Columbia University, the University of Miami, the Department of Veterans Affairs - Los Angeles and the University of Minnesota.

\section{Brain protein extraction}

Aqueous brain protein extracts were prepared based on a protocol previously published [34]. Specifically, human tissue specimens of $0.3-0.5 \mathrm{~g}$ were cut (or hemiforebrains of rTg4510 mice were dissected), transferred to 5 volumes ( $1 \mathrm{~g}$ per $5 \mathrm{~mL}$ ) of ice-cold extraction buffer (25 mM tris(hydroxymethyl)aminomethane-hydrochloric acid (Tris- $\mathrm{HCl}$ ), $\mathrm{pH} 7.4 ; 140 \mathrm{mM} \mathrm{NaCl}$ and $3 \mathrm{mM} \mathrm{KCl}$ with the following protease and phosphatase inhibitors: $0.1 \mathrm{mM}$ phenylmethylsulfonyl fluoride, $0.2 \mathrm{mM} 1,10$-phenanthroline monohydrate, a protease inhibitor cocktail (MilliporeSigma, Burlington, MA) and two phosphatase inhibitor cocktails (MilliporeSigma)), and homogenized using a Dounce homogenizer. The resulting material was centrifuged for $90 \mathrm{~min}\left(16,100 \mathrm{~g}, 4^{\circ} \mathrm{C}\right)$. The supernatant was subsequently depleted of endogenous immunoglobulin G (IgG) with Protein G Sepharose 4 Fast Flow beads (GE Healthcare, Piscataway, NJ; $50 \mu \mathrm{L}$ of slurry per $500 \mu \mathrm{L}$ of sample), and stored at $-20^{\circ} \mathrm{C}$. Protein concentrations of brain extracts were determined using a bicinchoninic acid protein assay kit (Thermo Scientific, Rockford, IL) according to the manufacturer's instructions.

\section{Immunoprecipitation}

To detect and measure levels of $\Delta$ tau314 and soluble tau proteins, $173 \mu \mathrm{g}$ of proteins from the aqueous brain extracts were brought to a volume of $500 \mu \mathrm{L}$ through the addition of immunoprecipitation (IP) dilution buffer (50 mM Tris$\mathrm{HCl}, \mathrm{pH} 7.4 ; 150 \mathrm{mM} \mathrm{NaCl}$ ) containing the protease and phosphatase inhibitors listed in Section Brain protein extraction. The resulting samples were incubated with $10 \mu \mathrm{g}$ of monoclonal antibody tau-13 (BioLegend, San Diego, CA) or mouse IgG (negative control). Immuno-complexes were captured by incubation with $30 \mu \mathrm{L}$ of Protein G Sepharose 4 Fast Flow resin at $4{ }^{\circ} \mathrm{C}$ for $14-16 \mathrm{~h}$. Following capture of immuno-complexes, resins were gently washed sequentially in IP buffer A (50 mM Tris- $\mathrm{HCl}, \mathrm{pH} 7.4 ; 300 \mathrm{mM} \mathrm{NaCl} ; 1$ $\mathrm{mM}$ ethylenediaminetetraacetic acid (EDTA) and $0.1 \%$ volume/volume (v/v) polyethylene glycol p-(1,1,3,3-tetramethylbutyl)-phenyl ether (Triton X-100) with protease and phosphatase inhibitors) and IP buffer B (50 mM Tris$\mathrm{HCl}, \mathrm{pH} 7.4 ; 150 \mathrm{mM} \mathrm{NaCl} ; 1 \mathrm{mM}$ EDTA and $0.1 \%$ (v/v) Triton X-100 with protease and phosphatase inhibitors) at $4{ }^{\circ} \mathrm{C}$ for $5 \mathrm{~min}$. Complexes were then eluted by boiling the magnetic beads at $95^{\circ} \mathrm{C}$ in $30 \mu \mathrm{L}$ of sodium dodecyl sulfate (SDS)-polyacrylamide gel electrophoresis (PAGE) loading buffer $(500 \mathrm{mM}$ Tris- $\mathrm{HCl}, \mathrm{pH} 8.0 ; 24 \%$ (v/v) glycerol; 8\% weight/volume (w/v) SDS; $0.01 \%(\mathrm{w} / \mathrm{v})$ Coomassie brilliant blue; $0.1 \%$ (v/v) phenol red; $710 \mathrm{mM} \beta$-mercaptoethanol); elutates were size-fractionated on $10 \%$ Criterion Tris- $\mathrm{HCl}$ Precast gels (Bio-Rad, Hercules, CA), and electrophoretically transferred onto $0.2-\mu \mathrm{m}$ nitrocellulose membranes at a constant current of $0.4 \mathrm{~A}$ for $4 \mathrm{~h}$ at $4{ }^{\circ} \mathrm{C}$.

\section{Western blotting}

Western blotting (WB) was performed according to a previously published protocol [21].

To measure levels of $\Delta$ tau314 and soluble total tau (T-tau) proteins, blots were probed with biotin-conjugated 4F3 $(1: 45,000$, final concentration $=36 \mathrm{ng} / \mathrm{mL})$ and biotinconjugated tau-5 (Thermo Fisher Scientific; 1:30,000, final concentration $=17 \mathrm{ng} / \mathrm{mL}$ ), respectively. Following $5 \mathrm{X} 5$-min washes in wash buffer $(10 \mathrm{mM}$ Tris- $\mathrm{HCl}, \mathrm{pH}$ 7.4; $200 \mathrm{mM} \mathrm{NaCl} ; 0.1 \%$ (v/v) polyoxyethylene (20) sorbitan monolaurate (Tween 20)), membranes were incubated at room temperature in horseradish peroxidase (HRP)-conjugated NeutrAvidin (Thermo Fisher Scientific; $1: 5,000$, final concentration $=0.2 \mu \mathrm{g} / \mathrm{mL}$ ) for $10 \mathrm{~min}$. The membranes were then washed again (5 X 5-min in wash buffer) prior to being developed.

To measure levels of Casp2, $100 \mu \mathrm{g}$ of proteins from the aqueous brain extracts were electrophoretically separated by SDS-PAGE using Criterion 10\% Tris- $\mathrm{HCl}$ Precast gels (Bio-Rad), and then transferred onto nitrocellulose membranes. Blots were probed with rabbit monoclonal anti-Casp2 (Abcam, Cambridge, MA; 1: 5,000, final concentration $=496 \mathrm{ng} / \mathrm{mL}$ ). Following 5 X 5-min washes in wash buffer, membranes were incubated at room temperature in HRP-conjugated anti-rabbit IgG (Thermo Fisher Scientific; 1:200,000, final concentration $=5 \mathrm{ng} / \mathrm{mL}$ ) for $1 \mathrm{~h}$. The membranes were then washed again (5 X 5min in wash buffer) prior to being developed.

To measure levels of glyceraldehyde 3-phosphate dehydrogenase (GAPDH), $20 \mu \mathrm{g}$ of proteins from the aqueous brain extracts were electrophoretically separated by SDSPAGE using Criterion 10\% Tris- $\mathrm{HCl}$ Precast gels (Bio-Rad), and then transferred onto nitrocellulose membranes. Blots were probed with rabbit monoclonal anti-GAPDH (Cell signaling Technology, Danvers, MA; 1: 4,000, final concentration $=25 \mathrm{ng} / \mathrm{mL}$ ). Following $5 \times 5$-min washes in wash buffer, membranes were incubated at room temperature in HRP-conjugated anti-rabbit IgG (Thermo Fisher Scientific; $1: 200,000$, final concentration $=5 \mathrm{ng} / \mathrm{mL}$ ) for $1 \mathrm{~h}$. The membranes were then washed again (5 X 5-min in wash buffer) prior to being developed.

Western blots were developed using the West Pico electrochemiluminescence detection system (Thermo Fisher Scientific) except for the blots probed with biotinconjugated 4F3 and anti-Casp2, which were developed using the West Femto detection system (Thermo Fisher Scientific). Signal intensities were quantified densitometrically using Optiquant (Packard Cyclone, Perkin-Elmer Life Sciences Inc., Boston, MA). 
Experimenters performing IP/WB were blind to the demographic and clinical characteristics of subjects. The levels of proteins were determined from two independent experiments, and the mean levels were used for statistical analysis.

\section{Statistical analysis}

Statistical analyses were performed using GraphPad Prism Version 7.04 (GraphPad Software, La Jolla, CA) and R Version 3.4.1 (R Foundation for Statistical Computing, Vienna, Austria). The demographic and neuropathologic characteristics were compared between HD patients and non-HD individuals using two-tailed, unpaired MannWhitney tests for continuous variables and two-sided Fisher's exact tests for binary variables. The associations between demographic characteristics and levels of $\Delta$ tau314 proteins (normalized to levels of T-tau proteins) were estimated using Spearman's rank-order correlations for continuous variables and Mann-Whitney tests for binary variables. Finally, levels of $\Delta$ tau314, T-tau, Casp2 and GAPDH were compared between HD patients and nonHD individuals using two-tailed, unpaired Mann-Whitney tests. Additionally, multiple linear regressions of the log transformed levels were fit with adjustment for the potential confounders of age at death, sex and PMI. The unadjusted version of this analysis-a two-tailed, unpaired $t$-test performed on the log transformed levels-is also presented. $P$ values of less than 0.05 were considered statistically significant.

\section{Results}

Levels of $\Delta$ tau314 proteins are elevated in the prefrontal cortex of HD patients in a small cohort

First, using a polymerase chain reaction (PCR)-based method previously published [40], we verified that the studied HD patients harbored > 35 CAG trinucleotide repeats in at least one allele, whereas the number of CAG repeats in the non-HD individuals did not exceed 35 in either allele (data not shown).

We then asked whether $\Delta$ tau314 proteins were present in the prefrontal cortex of studied subjects. For this, we used 4F3 (Table 1), a monoclonal antibody that specifically targets truncated tau proteins ending C-terminally at D314, to detect $\Delta$ tau314 proteins in the prefrontal cortex (BA 8) of five HD patients and five non-HD individuals, a cohort of the HUB-ICO-IDIBELL Biobank, Spain (Additional file 1: Table S1). Age at death, sex and PMI of brain tissue harvest in the HD patients and the non-HD individuals were matched (Additional file 1: Table S2).

We identified 4F3-reactive $\Delta$ tau314 proteins in all the studied subjects (Fig. 1a). The levels of $\Delta$ tau314 proteins were elevated 1.6-fold in the HD patients compared to the non-HD individuals (Fig. 1b, Additional file 1: Table S3), and trended higher following adjustments for age at death, sex and PMI of brain tissue harvest (Additional file 1: Table S3).

\section{Levels of $\Delta$ tau314 proteins are elevated in the prefrontal cortex of HD patients in a large cohort}

We then attempted to confirm these findings in a larger cohort of subjects. Based on the quantitative analysis of 4F3-reactive $\Delta$ tau314 proteins, we determined that the lowest sample size required for each group to achieve statistical significance under type I error $\alpha=0.05$ and power (i.e., $(1-\beta))=0.80$ is $N=11$.

We measured levels of $\Delta$ tau314 proteins in the prefrontal cortex (BA 8/9) of thirteen HD patients and fourteen non-HD individuals (Additional file 1: Table S1). Age at death, sex distribution and PMI of brain tissue harvest did not significantly differ between the HD patients and the non-HD individuals (Table 2). We found that levels of $\Delta$ tau314 proteins were 3.1 -fold higher in the HD patients than in the non-HD individuals (Fig. 1c and d, Table 3).

\section{Levels of $\Delta$ tau314 proteins are elevated in the caudate nucleus of HD patients in a large cohort}

Next, we measured levels of 4F3-reactive $\Delta$ tau314 proteins in the caudate nucleus, a severely affected region, in a subset of the large cohort - eleven HD patients and twelve non-HD individuals (Additional file 1: Table S1). Age at death, but not sex distribution or PMI of brain tissue harvest, was significantly different between the HD patients and the non-HD individuals (Table 4). We found that levels of $\Delta$ tau314 proteins in the caudate were 5.6-fold higher in HD patients than in non-HD individuals (Fig. 1e and f, Table 5).

Table 1 Antibodies used in this study

\begin{tabular}{|c|c|c|c|}
\hline Antibody & Host/Isotype & Epitope $^{a}$ & Source \\
\hline tau-13 & $M s, \lg G_{1, k}$ & $\mathrm{Tau}_{15-25}$ & BioLegend, Cat. \#: 835201 \\
\hline tau-5 (biotin-conjugated) & $M s, \operatorname{lgG}_{1}$ & $\operatorname{Tau}_{210-241}$ & Thermo Fisher Scientific, Cat. \#: MA5-12805 \\
\hline 4F3 (biotin-conjugated) & $M s, \lg G_{2 b, k}$ & $\operatorname{Tau}_{x-314}$ & Ashe laboratory \\
\hline Anti-Casp2 & $R b, \lg G$ & C-terminus & Abcam, Cat. \#: 179519 \\
\hline Anti-GAPDH & $\mathrm{Rb}, \lg G$ & C-terminus & Cell Signaling Technology, Cat. \#: 2118 \\
\hline
\end{tabular}

${ }^{\mathrm{a}}$ Amino acid residues of tau protein are counted using the $2 \mathrm{~N} 4 \mathrm{R}$ isoform numbering system 


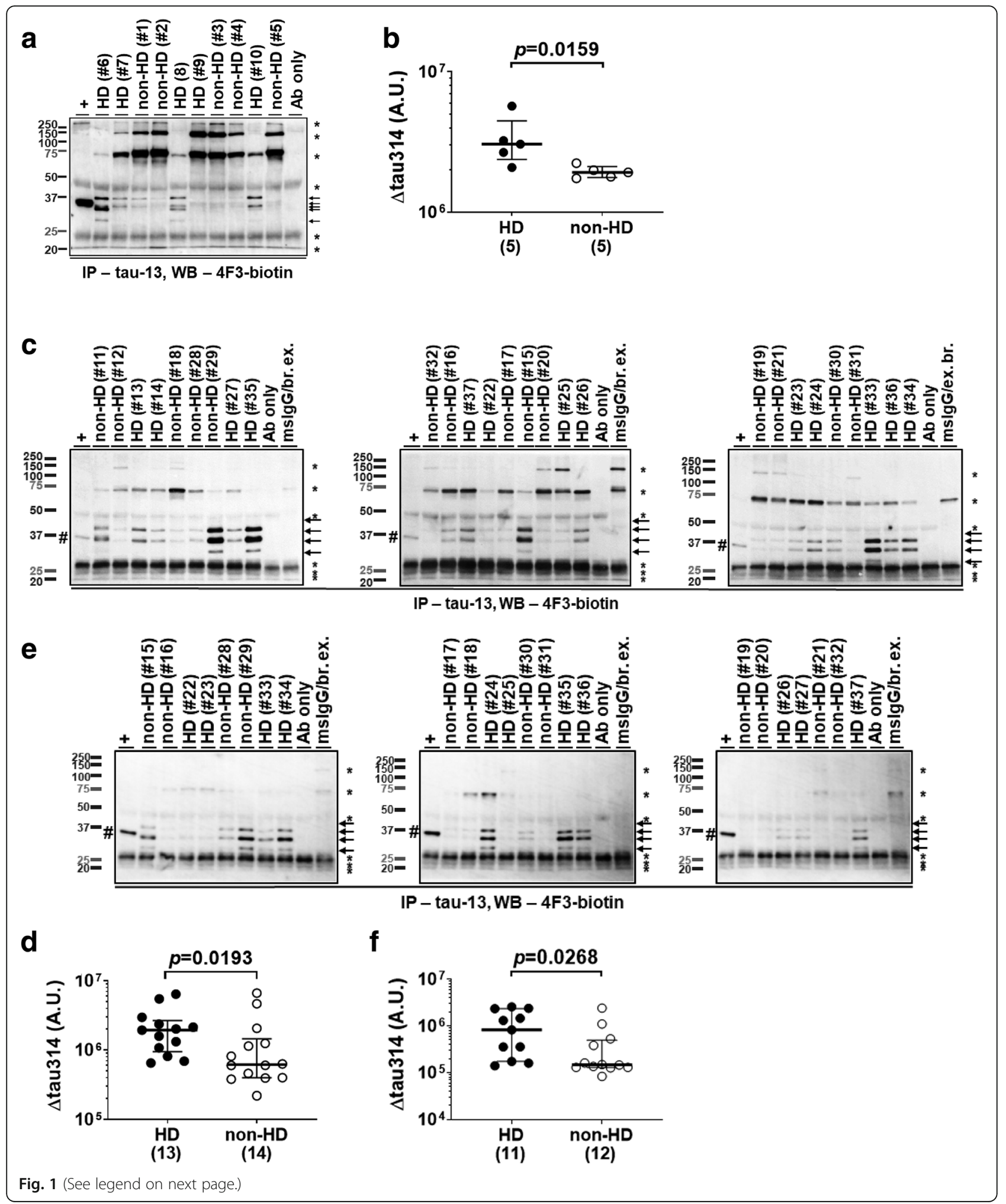


(See figure on previous page.)

Fig. 1 Levels of $\Delta$ tau314 proteins are elevated in HD patients. a, c, e Representative IP/WB showing that the tau-13-immunoprecipitated $\Delta$ tau314 proteins (arrows) were detected by the biotin-conjugated 4F3 antibody in the prefrontal cortex (Brodmann's area (BA) 8) of subjects from the HUB-ICO-IDIBELL Biobank, Spain (a), in the prefrontal cortex (BA 8/9) of subjects from the NIH NeuroBioBank and the New York Brain Bank (c), and in the caudate nucleus of subjects from the NIH NeuroBioBank (e). HD, Huntington's disease patients; non-HD, individuals without Huntington's disease. + , in vitro synthetic $\Delta$ tau314 proteins (hash; $20 \mu \mathrm{L}$ of sample used in IP, positive control). ${ }^{*}$, non-specific bands. Sample IDs and disease diagnoses (Additional file 1: Table S1) were shown for IP reactions. Ab only, IP reaction containing only $10 \mu \mathrm{g}$ of tau-13 (negative control). MslgG/ br. ex., IP reaction containing $10 \mu \mathrm{g}$ of mouse lgG and $173 \mu \mathrm{g}$ of soluble brain proteins (negative control). For the MslgG/br. ex. lanes in figure C, brain extracts were a mixture of the nine samples studied in the same blot with each contributing 1/9th the amount (i.e., $19.2 \mu \mathrm{g}$ ); for the MslgG/ br. ex. lanes in figure e, 1/8th the amount (i.e., $21.6 \mu \mathrm{g}$ ). $\mathbf{b}, \mathbf{d}$, $\mathbf{f}$ Comparison of levels of $\Delta$ tau314 proteins in the prefrontal cortex (BA 8) of HD patients and non-HD individuals from the HUB-ICO-IDIBELL Biobank, Spain (b), in the prefrontal cortex (BA 8/9), from the NIH NeuroBioBank and the New York Brain Bank (d), and in the caudate nucleus, from the NIH NeuroBioBank (f). The numbers of analyzed subjects are shown in parentheses. A.U. = arbitrary unit. The $y$-axes in figures are in log scale. Mann-Whitney test was used for between-group comparison; medians (middle long bars), and 1st (lower short bars) and 3rd (upper short bars) quartiles are shown

\section{Levels of soluble total tau proteins are lower in the prefrontal cortex and caudate nucleus of HD patients in the large cohort}

To ascertain whether the increases in $\Delta$ tau314 proteins was due to an increase in soluble total tau ( $\mathrm{T}$-tau) proteins, we measured the levels of T-tau proteins that were immunoprecipitated by tau- 13 antibody, and detected by tau-5, a monoclonal antibody directed against a midregional epitope of tau (Table 1). We quantified levels of T-tau proteins in the prefrontal cortex and caudate nucleus of subjects in the large cohort, and in the prefrontal cortex of subjects in the small cohort.

Interestingly, in the large cohort, levels of T-tau proteins in the prefrontal cortex of HD patients were $53 \%$ lower than in non-HD individuals (Fig. 2a and b, Table 3), indicating that the elevated levels of $\Delta$ tau314 proteins in HD patients were not due to an overall increase in T-tau proteins. The reduction in T-tau proteins in HD patients resulted in 5.3-fold increase in the ratio of $\Delta$ tau314:T-tau in HD patients versus non-HD individuals (Fig. 2c, Table 3).

Table 2 Comparison of demographic characteristics of HD patients and non-HD individuals from the NIH NeuroBioBank and the New York Brain Bank used in the study of proteins in the prefrontal cortex (BA8/9)

\begin{tabular}{llll}
\hline & non-HD & HD & p value \\
\hline $\begin{array}{l}\text { Sample size, } N \\
\text { Age at death [yr]: }\end{array}$ & 14 & 13 & \\
$\quad \begin{array}{l}\text { median (1st quartile, } \\
\text { 3rd quartile) }\end{array}$ & $71.5(61.5,79.5)$ & $65.0(60.0,72.0)$ & $0.12^{\mathrm{a}}$ \\
$\quad$ range & $57.0-89.0$ & $50.0-77.0$ & \\
$\begin{array}{l}\text { Sex, female/male, } \\
\text { No. (\% female) }\end{array}$ & $8 / 6(47.1 \%)$ & $9 / 4(52.9 \%)$ & $0.69^{\mathrm{b}}$ \\
$\begin{array}{l}\text { Post-mortem interval [hr]: } \\
\quad \text { median (1st quartile, }\end{array}$ & $12.6(11.2,18.1)$ & $12.0(9.2,17.2)$ & $0.68^{\mathrm{a}}$ \\
$\begin{array}{l}\text { 3rd quartile) } \\
\text { range }\end{array}$ & $9.0-20.5$ & $6.0-21.7$ & \\
\hline
\end{tabular}

aTwo-tailed, unpaired Mann-Whitney test

bTwo-sided Fisher's exact test
Similar to the prefrontal cortex, the levels of T-tau proteins in the caudate nucleus were $69 \%$ lower in HD patients than in non-HD individuals (Fig. 2d and e, Table 5). Normalizing $\Delta$ tau314 to T-tau proteins accentuated their elevated levels, resulting in 13.8fold higher levels in HD patients compared to the nonHD individuals (Fig. 2f, Table 5). As in the prefrontal cortex, the elevation of $\Delta$ tau314 proteins in the caudate nucleus of HD patients is not due to an overall increase in T-tau proteins.

In the small cohort, the levels of T-tau proteins were comparable between the HD patients and non-HD individuals (Fig. $2 \mathrm{~g}$ and $\mathrm{h}$, Additional file 1: Table S3). Our inability to detect a significant decrease in the level of $\mathrm{T}$-tau proteins in the small cohort is likely due to the small sample size $(N=5 /$ group $)$. Based on the quantitative analysis of tau 5 -reactive proteins in the prefrontal cortex of subjects in the large cohort, we determined that the lowest sample size required to achieve statistical significance under type I error $\alpha=0.05$ and power (i.e., $(1-\beta))=0.80$ is $N=82$.

To further investigating how levels of $\Delta$ tau314 proteins associate with levels of soluble T-tau proteins in the large cohort, we measured the levels of T-tau (T-tau (WB)) proteins revealed by direct WB probed with tau-5.

Table 3 A statistical comparison of protein levels in the prefrontal cortex (BA8/9) of HD patients and non-HD individuals from the NIH NeuroBioBank and the New York Brain Bank

\begin{tabular}{llll}
\hline & Mann-Whitney $^{\mathrm{a}}$ & t-test $^{\mathrm{b}}$ & Multiple linear regression $^{\mathrm{c}}$ \\
\hline$\Delta$ tau314 & $p=0.019$ & $p=0.035$ & $p=0.094$ \\
T-tau & $p=0.011$ & $p=0.075$ & $p=0.099$ \\
$\Delta$ tau314:T-tau & $p=0.00082$ & $p=0.013$ & $p=0.025$ \\
GAPDH & $p=0.46$ & $p=0.88$ & $p=0.35$ \\
Casp2:GAPDH & $p=0.0078$ & $p=0.0030$ & $p=0.019$
\end{tabular}

\section{${ }^{a}$ The two-tailed, unpaired Mann-Whitney test was performed}

'The two-tailed, unpaired $t$-test with Welch's correction was performed on the log transformed outcomes

${ }^{c}$ Multiple linear regression was used to analyze the log transformed outcomes of protein levels with adjustment for age at death, sex and PMI of brain tissue harvest 
Table 4 Comparison of demographic characteristics of HD patients and non-HD individuals from the NIH NeuroBioBank used in the study of proteins in caudate nucleus

\begin{tabular}{llll}
\hline & non-HD & HD & $p$ value \\
\hline Sample size, $N$ & 12 & 11 & \\
$\begin{array}{l}\text { Age at death [yr]: } \\
\quad \text { median (1st quartile, }\end{array}$ & $74.0(68.0,80.5)$ & $65.0(58.0,73.0)$ & $0.04^{\text {a }}$ \\
$\quad$ 3rd quartile) & & & \\
$\quad$ range & $60.0-89.0$ & $50.0-77.0$ & $>0.99^{b}$ \\
$\begin{array}{l}\text { Sex, female/male, } \\
\text { No. (\% female) }\end{array}$ & $7 / 5(58.3 \%)$ & $7 / 4(63.6 \%)$ & \\
$\begin{array}{l}\text { Post-mortem interval [hr]: } \\
\text { median (1st quartile, }\end{array}$ & $13.8(11.1,18.6)$ & $12.0(9.2,15.7)$ & $0.44^{\text {a }}$ \\
3rd quartile) & & & \\
range & $9.0-20.5$ & $8.6-21.7$ & \\
\hline
\end{tabular}

${ }^{a}$ Two-tailed, unpaired Mann-Whitney test

${ }^{\mathrm{b}}$ Two-sided Fisher's exact test

These tau-5-detected proteins are not necessarily tau13-immunoreactive. In the prefrontal cortex, levels of T-tau (WB) proteins in the HD patients were 34\% lower than in the non-HD individuals (Additional file 1: Figure S1a and S1b, Table S4). As a result, the ratio of $\Delta$ tau314:T-tau (WB) in HD patients was 3.8fold higher than in non-HD individuals (Additional file 1: Figure S2a, Table S4). Similarly, in the caudate nucleus, levels of the T-tau (WB) proteins were $44 \%$ lower in HD patients than in non-HD individuals (Additional file 1: Figure S1c and S1d, Table S5). Normalizing $\Delta$ tau314 to $\mathrm{T}$-tau (WB) proteins resulted in 21.9-fold higher levels in HD patients than in nonHD individuals (Additional file 1: Figure S2b, Table S5). These findings further support that the higher levels of $\Delta$ tau314 proteins in HD patients are not due to an increase in $\mathrm{T}$-tau proteins.

To confirm that our findings in levels of $\Delta$ tau314 and T-tau proteins are not due to difference in the amount of soluble brain proteins applied to IP/WB, we analyzed

Table 5 A statistical comparison of protein levels in the caudate nucleus of HD patients and non-HD individuals from the NIH NeuroBioBank

\begin{tabular}{llll}
\hline & Mann-Whitney $^{\mathrm{a}}$ & t-test $^{\mathrm{b}}$ & $\begin{array}{l}\text { Multiple linear } \\
\text { regression }\end{array}$ \\
\hline$\Delta$ tau314 & $p=0.027$ & $p=0.041$ & $p=0.082$ \\
T-tau & $p=0.00055$ & $p=0.0031$ & $p=0.038$ \\
$\Delta$ tau314:T-tau & $p=0.0013$ & $p=0.00064$ & $p=0.0041$ \\
GAPDH & $p=0.15$ & $p=0.29$ & $p=0.29$ \\
Casp2:GAPDH & $p=0.0036$ & $p=0.0035$ & $p=0.0070$ \\
\hline
\end{tabular}

${ }^{a}$ The two-tailed, unpaired Mann-Whitney test was performed

${ }^{\mathrm{b}}$ The two-tailed, unpaired $t$-test with Welch's correction was performed on the log transformed outcomes

'Multiple linear regression was used to analyze the log transformed outcomes of protein levels with adjustment for age at death, sex and PMI of brain tissue harvest levels of the housekeeping protein GAPDH. We showed that the levels of GAPDH were comparable between HD patients and non-HD individuals in both prefrontal cortex and caudate nucleus (Additional file 1: Figure S3, Tables 3 and 5).

Lastly, we examined the relationships between demographic characteristics of subjects and levels of $\Delta$ tau314 proteins in both the large and small cohorts. We found no apparent correlation of $\Delta$ tau314 protein levels (normalized to levels of T-tau) with either ages at death or PMIs of brain tissue harvest (Additional file 1: Figure S4a, S4b, S4d, S4e, S4g and S4h). Also, normalized $\Delta$ tau314 protein levels were comparable between female and male individuals (Additional file 1: Figure S4c, S4f and $\mathrm{S} 4 \mathrm{i}$ ).

\section{Levels of Casp2 are higher in the prefrontal cortex and caudate nucleus of HD patients}

To understand the extent to which Casp2 is involved in production of $\Delta$ tau314 proteins in HD, we measured levels of Casp2 detected by a monoclonal anti-Casp2 antibody directed against a C-terminal epitope (Table 1) in the prefrontal cortex and the caudate nucleus of subjects in the large cohort. Prior to the experiments, we validated the binding specificity of the anti-Casp2 antibody to the cleaved small subunit of recombinant Casp2 (data not shown).

In the prefrontal cortex, levels of Casp2 proteins, normalized to levels of the house-keeping protein GAPDH, were 1.7-fold higher in HD patients than in non-HD individuals (Fig. 3a and b, Table 3). In the caudate nucleus, levels of Casp2 proteins, normalized to levels of GAPDH, were 2.5-fold higher in HD patients than in non-HD individuals (Fig. 3d and e, Table 5). The finding that levels of both $\Delta$ tau314 and Casp2 proteins are higher in HD patients than in non-HD individuals, in two distinct HD-affected brain structures, supports the active involvement of Casp2 in $\Delta$ tau314 production in human subjects, particularly in HD patients.

In addition, levels of Casp2 (normalized to levels of GAPDH) correlated well with levels of $\Delta$ tau314 (normalized to levels of T-tau) in both the prefrontal cortex (Fig. 3c) and the caudate nucleus (Fig. 3f), which further supports an association between Casp2 and the production of $\Delta$ tau314 proteins.

\section{Discussion}

In this study, we focused on understanding the potential role of $\Delta$ tau314, a soluble, cognition-related tau protein species associated with Casp2, in HD. Using biochemical analyses, we detected $\Delta$ tau314 proteins in the striatum (caudate nucleus) and prefrontal cortex (BA 8/9), areas that are especially vulnerable to HD pathology. We showed that levels of $\Delta$ tau314 proteins are 1.6- to 5.6- 


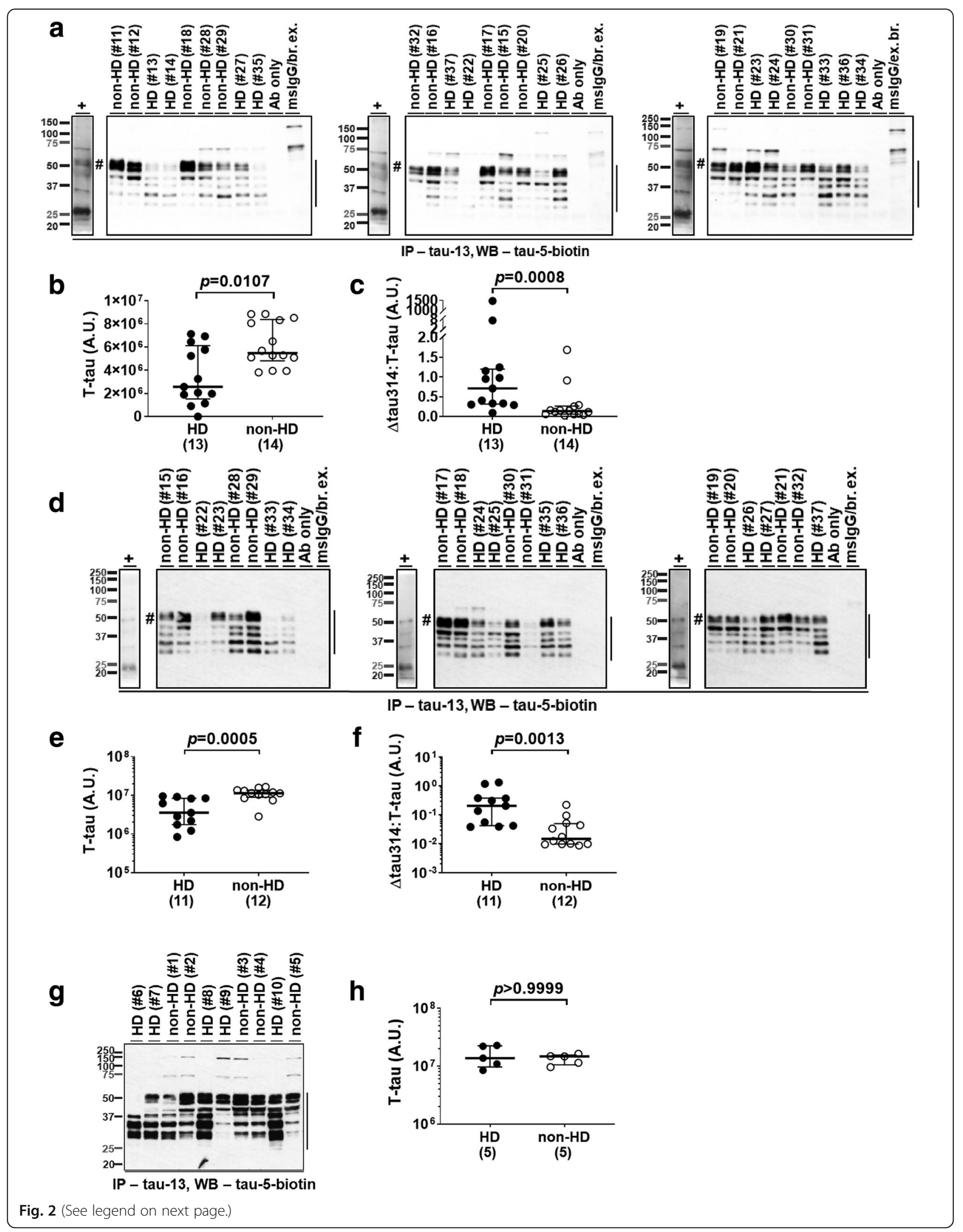




\section{(See figure on previous page.)}

Fig. 2 Levels of soluble total tau proteins are lowered in HD patients of the large, but not the small, cohort. a, d, $\mathbf{g}$ Representative IP/WB showing that the tau-13-immunoprecipitated soluble total tau (T-tau) proteins (vertical bars) were detected by the biotin-conjugated tau-5 antibody in the prefrontal cortex (Brodmann's area (BA) 8/9) of subjects from the NIH NeuroBioBank and the New York Brain Bank (a), in the caudate nucleus of subjects from the NIH NeuroBioBank (d), and in the prefrontal cortex (BA 8) of subjects from the HUB-ICO-IDIBELL Biobank, Spain (g). HD, Huntington's disease patients; non-HD, individuals without Huntington's disease. +, in vitro synthetic full-length tau protein (hash; $20 \mu \mathrm{L}$ of sample used in IP, positive control). Sample IDs and disease diagnoses (Additional file 1: Table S1) were shown for IP reactions. Ab only, IP reaction containing only $10 \mu \mathrm{g}$ of tau-13 (negative control). MslgG/br. ex., IP reaction containing $10 \mu \mathrm{g}$ of mouse $\operatorname{lgG}$ and $173 \mu \mathrm{g}$ of soluble brain proteins (negative control). For the MslgG/br. ex. lanes in figure a, brain extracts were a mixture of the nine samples studied in the same blot with each contributing 1/9th the amount (i.e., $19.2 \mu \mathrm{g}$ ); for the MslgG/br. ex. lanes in figure d, 1/8th the amount (i.e., $21.6 \mu \mathrm{g}$ ). $\mathbf{b}, \mathbf{e}, \mathbf{h}$ Comparison of levels of T-tau proteins in the prefrontal cortex (BA 8/9) of HD patients and non-HD individuals from the NIH NeuroBioBank and the New York Brain Bank (b), in the caudate nucleus, from the NIH NeuroBioBank (e), and in the prefrontal cortex (BA 8), from the HUB-ICOIDIBELL Biobank, Spain (h). c, f Comparison of levels of $\Delta$ tau314 proteins, normalized to levels of T-tau proteins, in the prefrontal cortex (BA 8/9) of HD patients and non-HD individuals from the NIH NeuroBioBank and the New York Brain Bank (c), and in the caudate nucleus, from the NIH NeuroBioBank (f). The numbers of analyzed subjects are shown in parentheses. A.U. = arbitrary unit. The $y$-axes in figures $e, f$ and $h$ are in log scale. Mann-Whitney test was used for between-group comparison; medians (middle long bars), and 1st (lower short bars) and 3rd (upper short bars) quartiles are shown

fold higher in both regions in HD patients compared to non-HD individuals, that levels of Casp2 are 1.7- to 2.5fold higher in HD patients than in non-HD individuals, and that levels of Casp2 and $\Delta$ tau314 proteins are correlated in both studied brain structures. Our results are supported by a previous finding that the immunostaining intensity of Casp2, which catalyzes $\Delta$ tau 314 production, is more intense in degenerating caudate neurons of HD patients than in those of age-matched controls [16]. Also of note, $\sim 35$ - and $\sim 40-\mathrm{kDa}$ tau protein species that match the migration pattern of some of the $\Delta$ tau314 proteins in SDS-PAGE show a remarkably higher level in the cortex of HD patients than control individuals [11], though their exact identities are unclear in that study.

We used IP/WB, rather than WB only, for identifying $\Delta$ tau314 in soluble human brain homogenates because of the enhanced sensitivity and specificity. Our choice of using monoclonal antibody tau-13 as the capture reagent for immunoprecipitation in this study are based on two considerations: 1) the sensitivity of tau-13, and 2) the relevance of measured proteins to cognitive dysfunction and dementia. Specifically, we tried several widely used, commercially available antibodies, including mouse monoclonal tau-5, rabbit polyclonal K9JA, in addition to tau-13, to immunoprecipitate tau proteins from soluble human brain homogenates. We found that tau13 produced the most sensitive results. For example, the $\sim 45-\mathrm{kDa} \Delta$ tau314 protein that is derived from the longest tau isoform (2N4R) can only be detected using tau-13 as the capture reagent. In addition, since Casp2-catalyzed production of the tau-13-immunoreactive $\Delta$ tau314 mediates synaptotoxicity in cellular models and cognition-impairing effects in mice, and levels of the tau13-immunoprecipitated $\Delta$ tau314 proteins are higher in subjects with mild cognitive impairment and $\mathrm{AD}$ than cognitively normal individuals [44], we focused on tau-13-immunoprecipitated $\Delta$ tau314 proteins for this study. It is possible that other $\Delta$ tau314 species exist in the brain, and that $\mathrm{N}$-terminally truncated $\Delta$ tau314 proteins may also contribute to the pathogenesis of neurodegenerative diseases.

$\Delta$ tau314 proteins are among the reported posttranslationally modified tau species whose levels are elevated in $\mathrm{AD}$ subjects, compared to healthy individuals, and correlate with the severity of cognitive dysfunction in a variety of mouse lines modeling frontotemporal dementia [24, 43, 44]. Recently, we found higher levels of $\Delta$ tau314 proteins in patients with Lewy body dementia than in non-demented Parkinson's disease patients (Smith et al. Acta Neuropathol. Commun. in press). These findings, taken with the current results, suggest that dementia may depend on Casp2-cleavage of tau in several neurodegenerative conditions.

We found elevated $\Delta$ tau314 protein levels not only in the prefrontal cortex but also in the caudate nucleus of HD patients, a brain structure known to be involved in motor control, as well as some forms of cognition. Thus, whether Casp2-mediated $\Delta$ tau314 production plays a role in a variety of behavioral abnormalities-both related and unrelated to cognition-in HD is an important question to be addressed in future studies.

Our findings of lower total tau levels in HD are superficially inconsistent with a previous finding of increased total tau levels in the frontal cortex of HD patients [11]. This discrepancy is likely due to different experimental methods in the two studies. Specifically, detergent-containing brain extracts were used in the previous study, while detergentfree, aqueous brain extracts were used in the present study. The detergent extracts likely contained solubilized tau aggregates, which would be absent in the aqueous extracts. In addition, monoclonal $5 \mathrm{E} 2$ antibody-reactive, $\sim 49-\mathrm{kDa}$ tau proteins were quantified in the previous study, whereas tau-13-immunoprecipitated, tau-5-reactive, 30-55-kDa tau proteins were quantified in this study.

The number of CAG repeats has profound implications in both clinical phenotype and pathologic characteristics 


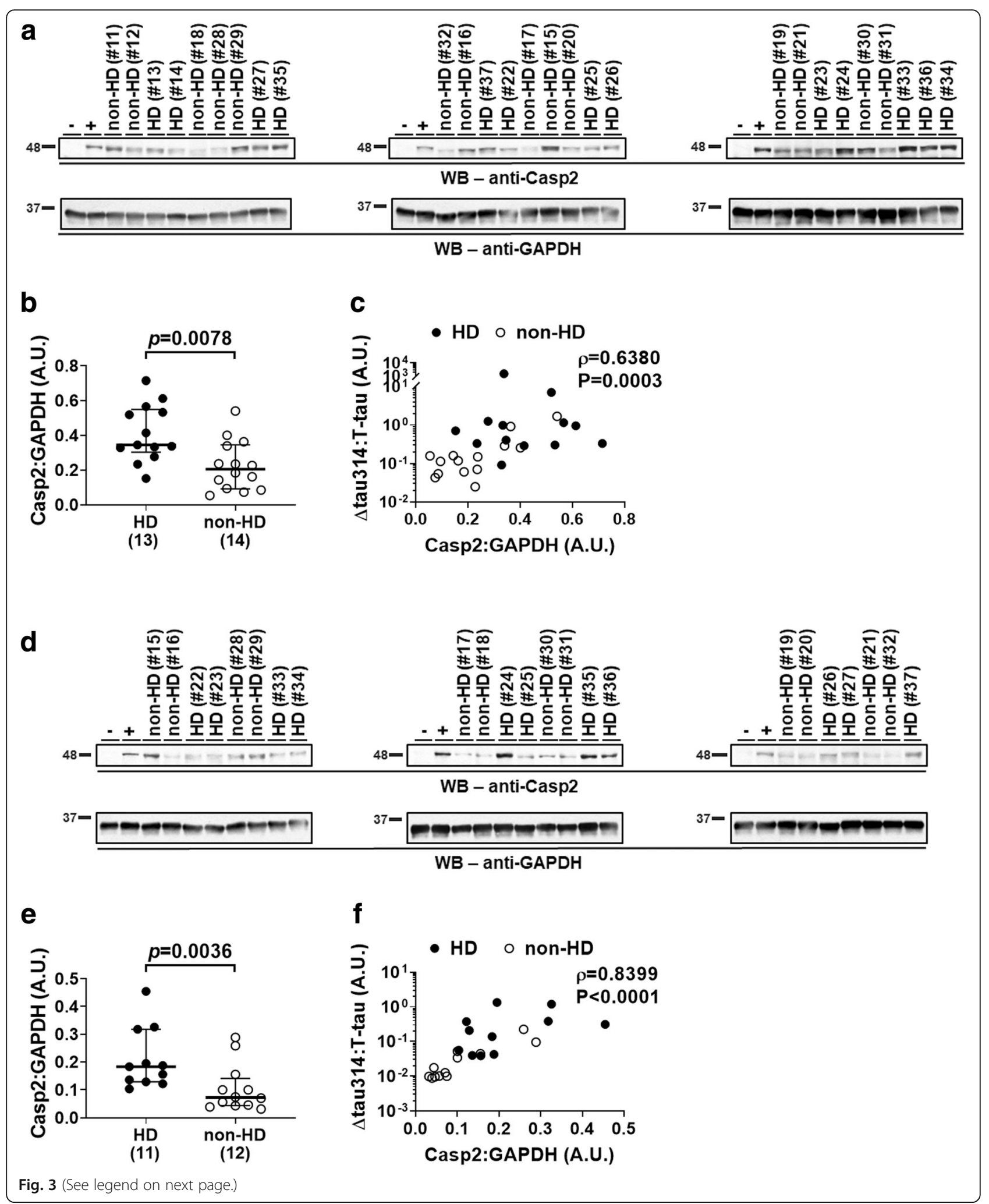


(See figure on previous page.)

Fig. 3 Levels of Casp2 are higher in HD patients of the large cohort. a, d Representative WB showing that Casp2 ( 48-kDa full-length form, upper panels) and GAPDH (loading control, lower panels; revealed in the same blots as Casp2) were detected in the prefrontal cortex (Brodmann's area (BA) 8/9) of subjects from the NIH NeuroBioBank and the New York Brain Bank (a), and in the caudate nucleus of subjects from the NIH NeuroBioBank (d). HD, Huntington's disease patients; non-HD, individuals without Huntington's disease. -, Casp2 knock-out FVB/129S6 mice (negative control); +, wild-type non-transgenic FVB/129S6 mice (positive control). Sample IDs and disease diagnoses (Additional file 1: Table S1) were shown. b, e Comparison of levels of Casp2 in the prefrontal cortex (BA 8/9) of HD patients and non-HD individuals from the NIH NeuroBioBank and the New York Brain Bank (b), and in the caudate nucleus, from the NIH NeuroBioBank (e). The numbers of analyzed subjects are shown in parentheses. Mann-Whitney test was used for between-group comparison; medians (middle long bars), and 1st (lower short bars) and 3rd (upper short bars) quartiles are shown. $\mathbf{c}$, $\mathbf{f}$ Correlation of levels of Casp2 (normalized to the house-keeping protein GAPDH) and levels

of $\Delta$ tau314 proteins (normalized to levels of T-tau proteins) in the prefrontal cortex (BA 8/9) of HD patients and non-HD individuals from the NIH NeuroBioBank and the New York Brain Bank (c), and in the caudate nucleus, from the NIH NeuroBioBank (f). Spearman's rank-order correlation was used. The $y$-axes in figures $c$ and $f$ are in $\log$ scale. A.U. = arbitrary unit

of HD [2, 29], and its relationship to the level of soluble $\Delta$ tau314 proteins warrants further investigation. Specifically, it would be interesting to extend the detection and quantitative analysis of $\Delta$ tau314 proteins to juvenile-onset HD patients, a population that often has significantly larger numbers of CAG repeats than the adult-onset patients and whose cognitive abilities are significantly impaired $[27,30]$. In this study, however, we chose to study HD subjects of 50 years or older at the age of death to avoid potential juvenile-onset HD-associated confounding factors since adult- and juvenile- onset HD patients manifest distinct clinical, pathologic and biochemical features $[4,5,37]$, suggesting different pathophysiological mechanisms through which mHTT exerts toxic effects.

Our finding of the association of HD with elevated levels of $\Delta$ tau314 supports the potential use of Casp2 inhibitors to ameliorate neurological dysfunction in HD. Broad-spectrum inhibitors of caspases were shown to reduce toxicity in cellular models of HD [1, $20,42]$, and ameliorate behavioral abnormalities in HD mice [28], indicating the disease-modifying effects of pharmacological blockage of caspase activities. In addition, using in silico structure-based design coupled with solid-state synthesis, a group of pentapeptide aldehyde compounds were recently developed, and some showed high affinity $\left(\mathrm{IC}_{50}\right.$ in nanomolar magnitude) and selectivity (60-fold difference in $\mathrm{IC}_{50}$ between Casp2 and caspase-3) to Casp2 [22], drawing attention to the possibility of targeting Casp2 activity as a therapeutic strategy in HD.

There are several limitations to this study. First, we did not determine the precise numbers of CAG repeats in the subjects, and thus the relationship between the degree of CAG expansion and levels of $\Delta$ tau314 (and Ttau) proteins is unknown. Second, to better understand the association of $\Delta$ tau314 proteins with other neurological functions, it will be necessary to perform biochemical analyses of $\Delta$ tau314 proteins in other brain structures (e.g., hippocampus and cerebellum). Third, an important question concerns the extent to which levels of $\Delta$ tau314 proteins correlate with the severity of cognitive dysfunction. For the subjects of the large cohort study, eight of the thirteen HD patients had cognition assessment records, and all demonstrated cognitive deficits prior to death. Meanwhile, four of the fourteen non-HD individuals had cognition assessment records, and all demonstrated normal cognitive function in their last assessment (Additional file 1: Table S1). Despite these observations, we did not perform a comprehensive evaluation of the correlation between levels of $\Delta$ tau314 proteins and the severity of cognitive deficits, because cognitive function assessments were lacking or not quantitatively provided for the other subjects, and different assessment approaches were used for those subjects whose cognitive functions were quantitatively measured. This is a question that may be addressed in humans and in animal models of HD in future studies. Fourth, the Casp2 protein band that we quantified using WB does not represent the fully active form. We identified only the $\sim 48-\mathrm{kDa}$ protein band, likely representing the procaspase-2 form, which, upon dimerization, shows a partial catalytic activity [3]. The fact that no cleaved small subunit of Casp2 was detected in the studied brains suggests that the fully active Casp2 may constitute a minute portion of the total pool of Casp2, and levels of the fully active Casp2 are below the limit of detection of WB. To the authors' knowledge, assays that specifically measure Casp2 activity in the brain are not yet available; development of such an assay is a priority of our future studies.

\section{Conclusions}

In conclusion, $\Delta$ tau314 proteins are present in the prefrontal cortex and caudate nucleus of human subjects, levels of Casp2 and $\Delta$ tau314 proteins are correlated, and levels of both Casp2 and $\Delta$ tau314 proteins are elevated in HD patients, compared to non-HD individuals. Findings of this study extend our understanding of the contribution of Casp2-mediated tau cleavage to HD pathogenesis, and support the development and exploration of Casp2 inhibitors in the treatment of HD. 


\section{Additional file}

Additional file 1: Figure S1. Levels of tau-5-reactive soluble proteins revealed by direct Western blotting are lowered in HD patients than nonHD individuals. Figure S2. Levels of tau-13-immunoreactive $\Delta$ tau314 proteins, following normalization to levels of tau-5-reactive proteins ( $T$ tau (WB)) revealed by direct Western blotting, are higher in HD patients than non-HD individuals. Figure S3. Levels of glyceraldehyde 3phosphate dehydrogenase (GAPDH) are comparable between HD patients and non-HD individuals of the large cohort. Figure S4. The relationships of $\Delta$ tau314 protein levels with demographic characteristics of subjects. Table S1. Demographic and neuropathological characteristics of human subjects. Table S2. Comparison of demographic characteristics of HD patients and non-HD individuals from the HUB-ICO-IDIBELL Biobank, Spain used in the study of proteins in the prefrontal cortex (BA8). Table S3. A statistical comparison of protein levels of HD patients and non-HD individuals from the HUB-ICO-IDIBELL Biobank, Spain. Table S4. A statistical comparison of levels of proteins revealed by direct Western blotting (WB) probed with tau-5 antibody in the prefrontal cortex (BA8/9) of HD patients and non-HD individuals from the NIH NeuroBioBank and the New York Brain Bank. Table S5. A statistical comparison of protein levels of proteins revealed by direct Western blotting (WB) probed with tau- 5 antibody in the caudate nucleus of HD patients and non$\mathrm{HD}$ individuals from the NIH NeuroBioBank. File S1 Supplementary references. (ZIP $8370 \mathrm{~kb}$ )

\section{Abbreviations}

ON4R: (microtubule-associated protein tau isoform containing) none $\mathrm{N}$ terminal inserts encoded by exons 2 and 3 and four microtubule-binding repeats; 2N4R: (microtubule-associated protein tau isoform containing) two $\mathrm{N}$ terminal inserts encoded by exons 2 and 3 and four microtubule-binding repeats; AD: Alzheimer's disease; BA: Brodmann's area; Casp2: Caspase-2; caspase: Cysteine-dependent aspartate-directed protease: cDNA: Complementary DNA; D314: aspartate being the 314th amino acid residue of a protein/polypeptide; EDTA: Ethylenediaminetetraacetic acid; GAPDH: Glyceraldehyde 3-phosphate dehydrogenase; HD: Huntington's disease; HRP: Horseradish peroxidase; HTT: Huntingtin; IC $\mathrm{C}_{50}$ : Half maximal inhibitory concentration; IgG : Immunoglobulin G; IP: Immunoprecipitation; IRBs: Institutional Review Boards; IT15: Interesting transcript 15; kDa: kiloDalton; MAPT: Human microtubule-associated protein tau gene; MAPT: Microtubule-associated protein tau; Mapt: Mouse microtubuleassociated protein tau gene; MCl: Mild cognitive impairment; $\mathrm{mHTT}$ : Mutan huntingtin; NIH: National Institutes of Health; NYBB: New York Brain Bank; PAGE: Polyacrylamide gel electrophoresis; PCR: Polymerase chain reaction; PMI: Post-mortem interval; rTg4510: transgenic mouse line 129S6.CgTg(Camk2a-tTA)1Mmay/JlwsJ; Fgf14Tg(tetO-MAPT*P301L)4510Kha/J, which overexpresses human microtubule-associated protein tau with the P301L mutation associated with frontotemporal dementia with parkinsonism linked to chromosome 17; SDS: Sodium dodecyl sulfate; Tris-

$\mathrm{HCl}$ : Tris(hydroxymethyl)aminomethane-hydrochloric acid; Triton X100: polyethylene glycol p-(1,1,3,3-tetramethylbutyl)-phenyl ether; Ttau: soluble total tau that is immunoprecipitated by the monoclonal antibody tau-13 directed against tau a.a. 15-25 (tau 2N4R numbering system) and immunoreactive to the monoclonal antibody tau-5 directed against tau a.a. 210-241 (tau 2N4R numbering system); Tween 20: polyoxyethylene (20) sorbitan monolaurate; v/v: volume/volume; w/v: Weight/volume; WB: Western blotting; $\Delta$ tau314: a C-terminally truncated tau protein ending at the 314th amino acid residue (aspartate) of the longest human tau splicing isoform

\section{Acknowledgements}

The authors thank the NIH NeuroBioBank, the New York Brain Bank and Dr. Isidre Ferrer at the HUB-ICO-IDIBELL Biobank, Spain for providing human brain specimens. We also thank Dr. Dennis J. Thiele at the Duke University for facilitating the acquisition of these human brain specimens.

\section{Authors' contributions}

PL, RG-P and KHA conceived the experiments. PL, BRS, ESH and AM performed the experiments and collected data. PL, BRS, AJP, RG-P and KHA analyzed and interpreted data. RG-P and JPGV provided key research materials. PL wrote the manuscript, and all authors contributed to editing and approving the manuscript.

\section{Funding}

This study, including experimental design, data collection, analysis and interpretation, and manuscript preparation, is supported by Huntington's Disease Society of America, Human Biology Project to R.G.-P.

\section{Availability of data and materials}

All data generated or analyzed during this study are included in this published article and its supplementary information files.

\section{Ethics approval and consent to participate}

Informed consent for clinical and neuropathologic evaluation and autopsy was obtained from all individual participants. All procedures of using deidentified human brain tissue, obtained at autopsy, were reviewed and approved by the Institutional Review Boards (IRBs) of HUB-ICO-IDIBELL Biobank (Spain), the Columbia University, the University of Miami, the Department of Veterans Affairs - Los Angeles and the University of Minnesota.

\section{Consent for publication}

Not applicable.

\section{Competing interests}

The authors declare that they have no competing interests.

\section{Author details}

${ }^{1}$ Departments of Neurology, University of Minnesota, Minneapolis, MN 55455 USA. ²Departments of Neuroscience, University of Minnesota, Minneapolis, MN 55455, USA. ${ }^{3} \mathrm{~N}$. Bud Grossman Center for Memory Research and Care, University of Minnesota, Minneapolis, MN 55455, USA. ${ }^{4}$ Division of Biostatistics, University of Minnesota, Minneapolis, MN 55455, USA. ${ }^{5}$ Wayzata High School, Plymouth, MN 55446, USA. ${ }^{6}$ Department of Pathology, Presbyterian Hospital and Columbia University, New York City, NY 10032, USA. ${ }^{7}$ Geriatric Research, Education, and Clinical Centers, Veterans Affairs Medical Center, Minneapolis, MN 55417, USA. ${ }^{8}$ Current address: University of Miami, Miller School of Medicine, Miami, FL 33136, USA.

Received: 16 May 2019 Accepted: 1 July 2019

Published online: 30 July 2019

\section{References}

1. Aiken CT, Tobin AJ, Schweitzer ES (2004) A cell-based screen for drugs to treat Huntington's disease. Neurobiol Dis 16:546-555. https://doi.org/10.1 016/j.nbd.2004.04.001

2. Andrew SE, Goldberg YP, Kremer B, Telenius H, Theilmann J, Adam S, Starr E, Squitieri F, Lin B, Kalchman MA et al (1993) The relationship between trinucleotide (CAG) repeat length and clinical features of Huntington's disease. Nat Genet 4:398-403. https://doi.org/10.1038/ng0893-398

3. Baliga BC, Read SH, Kumar S (2004) The biochemical mechanism of caspase2 activation. Cell Death Differ 11:1234-1241. https://doi.org/10.1038/sj. cdd.4401492

4. Bird ED, Iversen LL (1974) Huntington's chorea. Post-mortem measurement of glutamic acid decarboxylase, choline acetyltransferase and dopamine in basal ganglia. Brain 97:457-472

5. Bittenbender JB, Quadfasel FA (1962) Rigid and akinetic forms of Huntington's chorea. Arch Neurol 7:275-288

6. Blum D, Herrera F, Francelle L, Mendes T, Basquin M, Obriot H, Demeyer D, Sergeant N, Gerhardt E, Brouillet E et al (2015) Mutant huntingtin alters tau phosphorylation and subcellular distribution. Hum Mol Genet 24:76-85. https://doi.org/10.1093/hmg/ddu421

7. Caparros-Lefebvre D, Kerdraon O, Devos D, Dhaenens CM, Blum D, Maurage CA, Delacourte A, Sablonniere B (2009) Association of corticobasal degeneration and Huntington's disease: can tau aggregates protect huntingtin toxicity? Mov Disord 24:1089-1090. https://doi.org/10.1002/ mds. 22204

8. Carroll JB, Southwell AL, Graham RK, Lerch JP, Ehrnhoefer DE, Cao LP, Zhang WN, Deng Y, Bissada N, Henkelman RM et al (2011) Mice lacking caspase-2 are protected from behavioral changes, but not pathology, in the YAC128 model of Huntington disease. Mol Neurodegener 6:59. https://doi.org/10.11 86/1750-1326-6-59 
9. Davis MY, Keene CD, Jayadev S, Bird T (2014) The co-occurrence of Alzheimer's disease and Huntington's disease: a neuropathological study of 15 elderly Huntington's disease subjects. J Huntingtons Dis 3:209-217. https://doi.org/10.3233/JHD-140111

10. DiFiglia M, Sapp E, Chase KO, Davies SW, Bates GP, Vonsattel JP, Aronin N (1997) Aggregation of huntingtin in neuronal intranuclear inclusions and dystrophic neurites in brain. Science 277:1990-1993

11. Fernandez-Nogales M, Cabrera JR, Santos-Galindo M, Hoozemans JJ, Ferrer I, Rozemuller AJ, Hernandez F, Avila J, Lucas JJ (2014) Huntington's disease is a four-repeat tauopathy with tau nuclear rods. Nat Med 20:881-885. https:// doi.org/10.1038/nm.3617

12. Graham RK, Deng Y, Slow EJ, Haigh B, Bissada N, Lu G, Pearson J, Shehadeh J, Bertram L, Murphy Z et al (2006) Cleavage at the caspase- 6 site is required for neuronal dysfunction and degeneration due to mutant huntingtin. Cell 125:1179-1191. https://doi.org/10.1016/j.cell.2006.04.026

13. Gratuze M, Noel A, Julien C, Cisbani G, Milot-Rousseau P, Morin F, Dickler M, Goupil C, Bezeau F, Poitras I et al (2015) Tau hyperphosphorylation and deregulation of calcineurin in mouse models of Huntington's disease. Hum Mol Genet 24:86-99. https://doi.org/10.1093/hmg/ddu456

14. Gutekunst CA, Li SH, Yi H, Mulroy JS, Kuemmerle S, Jones R, Rye D, Ferrante RJ, Hersch SM, Li XJ (1999) Nuclear and neuropil aggregates in Huntington's disease: relationship to neuropathology. J Neurosci 19:2522-2534

15. HDCRG (1993) A novel gene containing a trinucleotide repeat that is expanded and unstable on Huntington's disease chromosomes. The Huntington's Disease Collaborative Research Group. Cell 72:971-983

16. Hermel E, Gafni J, Propp SS, Leavitt BR, Wellington CL, Young JE, Hackam AS, Logvinova AV, Peel AL, Chen SF et al (2004) Specific caspase interactions and amplification are involved in selective neuronal vulnerability in Huntington's disease. Cell Death Differ 11:424-438. https://doi.org/10.1038/ s..cdd. 4401358

17. Jellinger KA (1998) Alzheimer-type lesions in Huntington's disease. J Neural Transm (Vienna) 105:787-799. https://doi.org/10.1007/s007020050095

18. Jimenez-Sanchez M, Licitra F, Underwood BR, Rubinsztein DC (2017) Huntington's disease: mechanisms of pathogenesis and therapeutic strategies. Cold Spring Harb Perspect Med 7. https://doi.org/10.1101/ cshperspect.a024240

19. Kim YJ, Yi Y, Sapp E, Wang Y, Cuiffo B, Kegel KB, Qin ZH, Aronin N, DiFiglia M (2001) Caspase 3-cleaved N-terminal fragments of wild-type and mutant huntingtin are present in normal and Huntington's disease brains, associate with membranes, and undergo calpain-dependent proteolysis. Proc Natl Acad Sci U S A 98:12784-12789. https://doi.org/10.1073/pnas.221451398

20. Leyva MJ, Degiacomo F, Kaltenbach LS, Holcomb J, Zhang N, Gafni J, Park H, Lo DC, Salvesen GS, Ellerby LM et al (2010) Identification and evaluation of small molecule pan-caspase inhibitors in Huntington's disease models. Chem Biol 17:1189-1200. https://doi.org/10.1016/j.chembiol.2010.08.014

21. Liu P, Kemper L, Wang J, Zahs KR, Ashe KH, Pasinetti GM (2011) Grape seed polyphenolic extract specifically decreases abeta*56 in the brains of $\operatorname{Tg} 2576$ mice. J Alzheimers Dis 26:657-666. https://doi.org/10.3233/JAD-2011-110383

22. Maillard MC, Brookfield FA, Courtney SM, Eustache FM, Gemkow MJ, Handel RK, Johnson LC, Johnson PD, Kerry MA, Krieger F et al (2011) Exploiting differences in caspase-2 and -3 S(2) subsites for selectivity: structure-based design, solid-phase synthesis and in vitro activity of novel substrate-based caspase-2 inhibitors. Bioorg Med Chem 19:5833-5851. https://doi.org/10.1 016/j.bmc.2011.08.020

23. McIntosh GC, Jameson HD, Markesbery WR (1978) Huntington disease associated with Alzheimer disease. Ann Neurol 3:545-548. https://doi.org/1 0.1002/ana.410030616

24. Min SW, Chen X, Tracy TE, Li Y, Zhou Y, Wang C, Shirakawa K, Minami SS, Defensor E, Mok SA et al (2015) Critical role of acetylation in tau-mediated neurodegeneration and cognitive deficits. Nat Med 21:1154-1162. https:// doi.org/10.1038/nm.3951

25. Moss RJ, Mastri AR, Schut $L J$ (1988) The coexistence and differentiation of late onset Huntington's disease and Alzheimer's disease. A case report and review of the literature. J Am Geriatr Soc 36:237-241

26. Myers RH, Sax DS, Schoenfeld M, Bird ED, Wolf PA, Vonsattel JP, White RF, Martin JB (1985) Late onset of Huntington's disease. J Neurol Neurosurg Psychiatry 48:530-534

27. Nance MA, Myers RH (2001) Juvenile onset Huntington's disease_clinical and research perspectives. Ment Retard Dev Disabil Res Rev 7:153-157. https://doi.org/10.1002/mrdd.1022
28. Ona VO, Li M, Vonsattel JP, Andrews LJ, Khan SQ, Chung WM, Frey AS, Menon AS, Li XJ, Stieg PE et al (1999) Inhibition of caspase-1 slows disease progression in a mouse model of Huntington's disease. Nature 399:263-267. https://doi.org/10.1038/20446

29. Penney JB Jr, Vonsattel JP, MacDonald ME, Gusella JF, Myers RH (1997) CAG repeat number governs the development rate of pathology in Huntington's disease. Ann Neurol 41:689-692. https://doi.org/10.1002/ana.410410521

30. Quarrell OWJ (2014) Juvenile Huntington's Disease. In: Bates G, Tabrizi S, Jones L, editors. Huntington's Disease. 4th ed. Oxford, New York: Oxford University Press; p. 66-85

31. Reyes MG, Gibbons S (1985) Dementia of the Alzheimer's type and Huntington's disease. Neurology 35:273-277

32. Roberson ED, Scearce-Levie $\mathrm{K}$, Palop JJ, Yan F, Cheng IH, Wu T, Gerstein H, Yu GQ, Mucke $L$ (2007) Reducing endogenous tau ameliorates amyloid beta-induced deficits in an Alzheimer's disease mouse model. Science 316: 750-754. https://doi.org/10.1126/science.1141736

33. Rub U, Vonsattel JP, Heinsen H, Korf HW (2015) The neuropathology of Huntington s disease: classical findings, recent developments and correlation to functional neuroanatomy. Adv Anat Embryol Cell Biol 217:1-146

34. Shankar GM, Li S, Mehta TH, Garcia-Munoz A, Shepardson NE, Smith I, Brett FM, Farrell MA, Rowan MJ, Lemere CA et al (2008) Amyloid-beta protein dimers isolated directly from Alzheimer's brains impair synaptic plasticity and memory. Nat Med 14:837-842. https://doi.org/10.1038/nm1782

35. Tracy TE, Sohn PD, Minami SS, Wang C, Min SW, Li Y, Zhou Y, Le D, Lo I, Ponnusamy R et al (2016) Acetylated tau obstructs KIBRA-mediated signaling in synaptic plasticity and promotes Tauopathy-related memory loss. Neuron 90:245-260. https://doi.org/10.1016/j.neuron.2016.03.005

36. Troy CM, Shelanski ML (2016) Caspase-2 and tau-a toxic partnership? Nat Med 22:1207-1208. https://doi.org/10.1038/nm.4227

37. Vonsattel JP, Keller C, Cortes Ramirez EP (2011) Huntington's disease neuropathology. Handb Clin Neurol 100:83-100. https://doi.org/10.1016/ B978-0-444-52014-2.00004-5

38. Vuono R, Winder-Rhodes S, de Silva R, Cisbani G, Drouin-Ouellet J, Network RlotEHsD, Spillantini MG, Cicchetti F, Barker RA (2015) The role of tau in the pathological process and clinical expression of Huntington's disease. Brain 138:1907-1918. https://doi.org/10.1093/brain/awv107

39. Walker FO (2007) Huntington's disease. Lancet 369:218-228. https://doi. org/10.1016/S0140-6736(07)60111-1

40. Warner JP, Barron LH, Brock DJ (1993) A new polymerase chain reaction (PCR) assay for the trinucleotide repeat that is unstable and expanded on Huntington's disease chromosomes. Mol Cell Probes 7:235-239. https://doi. org/10.1006/mcpr.1993.1034

41. Wellington CL, Ellerby LM, Hackam AS, Margolis RL, Trifiro MA, Singaraja R, McCutcheon K, Salvesen GS, Propp SS, Bromm M et al (1998) Caspase cleavage of gene products associated with triplet expansion disorders generates truncated fragments containing the polyglutamine tract. J Biol Chem 273:9158-9167

42. Wellington $\mathrm{CL}$, Singaraja R, Ellerby $\mathrm{L}$, Savill J, Roy $\mathrm{S}$, Leavitt $\mathrm{B}$, Cattaneo $\mathrm{E}$, Hackam A, Sharp A, Thornberry N et al (2000) Inhibiting caspase cleavage of huntingtin reduces toxicity and aggregate formation in neuronal and nonneuronal cells. J Biol Chem 275:19831-19838. https://doi.org/10.1074/ jbc.M001475200

43. Zhang Z, Song M, Liu X, Kang SS, Kwon IS, Duong DM, Seyfried NT, Hu WT, Liu Z, Wang JZ et al (2014) Cleavage of tau by asparagine endopeptidase mediates the neurofibrillary pathology in Alzheimer's disease. Nat Med 20: 1254-1262. https://doi.org/10.1038/nm.3700

44. Zhao X, Kotilinek LA, Smith B, Hlynialuk C, Zahs K, Ramsden M, Cleary J, Ashe KH (2016) Caspase-2 cleavage of tau reversibly impairs memory. Nat Med 22:1268-1276. https://doi.org/10.1038/nm.4199

\section{Publisher's Note}

Springer Nature remains neutral with regard to jurisdictional claims in published maps and institutional affiliations. 